\title{
Isoform-specific alanine aminotransferase measurement can distinguish hepatic from extrahepatic injury in humans
}

\author{
INGALILL RAFTER ${ }^{1}$, TRULS GRÅBERG ${ }^{2}$, ANNA KOTRONEN $^{3}$, LOVISA STRÖMMER $^{2}$, C. MIKAEL MATTSON $^{4}$, \\ RAY W. KIM ${ }^{5}$, EWA EHRENBORG ${ }^{6}$, HÅKAN B. ANDERSSON ${ }^{1}$, HANNELE YKI-JÄRVINEN ${ }^{3}$, \\ INA SCHUPPE-KOISTINEN ${ }^{1}$, BJÖRN EKBLOM ${ }^{4}$, IAN COTGREAVE ${ }^{1}$ and BJÖRN GLINGHAMMAR ${ }^{1}$ \\ ${ }^{1}$ Safety Assessment, AstraZeneca, Södertälje; ${ }^{2}$ Department of Clinical Science, Intervention and Technology (CLINTEC), \\ Division of Surgery, Karolinska Institute at Karolinska University Hospital, Huddinge, Stockholm, Sweden; \\ ${ }^{3}$ Department of Medicine, University of Helsinki, Helsinki, Finland; ${ }^{4}$ Åstrand Laboratory of Work Physiology, \\ Swedish School of Sport and Health Sciences, Karolinska Institute, Stockholm, Sweden; \\ ${ }^{5}$ Division of Gastroenterology and Hepatology, Mayo Clinic, Rochester, MN, USA; \\ ${ }^{6}$ Department of Medicine, Center for Molecular Medicine, Karolinska Institute, Stockholm, Sweden
}

Received May 15, 2012; Accepted June 13, 2012

DOI: $10.3892 /$ ijmm.2012.1106

\begin{abstract}
Serum alanine aminotransferase (ALT) is used as a clinical marker to detect hepatic damage and hepatoxicity. Two isoforms of ALT have been identified, ALT1 and ALT2, which have identical enzymatic capacities and are detected simultaneously in human serum/plasma using classical clinical chemical assays. Differences exist in the expression patterns of the ALT1 and ALT2 proteins in different organs which suggest that changes in the proportion of ALT1 and ALT2 in plasma may arise and reflect damage to different human organs. However, this has not been previously studied due to the lack of a selective methodology that can quantify both ALT1 and ALT2 isoforms in the total ALT activity normally measured in clinical samples. To the best of our knowledge, our current study reveals for the first time, that under 3 different conditions of liver damage (non-alcoholic fatty liver disease, hepatitis $\mathrm{C}$ and during liver surgery) the leakage of ALT1 activity into plasma greatly exceeds that of ALT2, and that the measurement of ALT1 during liver damage is equal to the measurement of total ALT activity. By contrast, during skeletal muscle injury, induced in volunteers by physical exertion, the leakage of ALT2 exceeds that of ALT1 and the proportion of circulating ALT isoforms changes accordingly. The ALT isoform changes occurring in plasma reflect previously demonstrated relative contents of ALT1 and ALT2 activities in human liver and skeletal muscle. These data suggest that assessing the percentage contribution of ALT1 and ALT2 activities to total ALT activity
\end{abstract}

Correspondence to: Dr Björn Glinghammar, Safety Assessment, AstraZeneca, S-151 85 Södertälje, Sweden

E-mail: bjorn.glinghammar@astrazeneca.com

Key words: alanine aminotransferase, liver injury, biomarkers, skeletal muscle injury, creatine kinase in plasma may distinguish hepatic from extrahepatic injury using the same standard analytical platform.

\section{Introduction}

Karmen et al (1) discovered in 1954 that the enzymatic activity of glutamic pyruvic transaminase was present in normal human blood and patients with different diseases had several fold higher activity of this enzyme. Since then, the more commonly used name is alanine aminotransferase (ALT) and its activity in serum has become the standard which is routinely used for the diagnosis and follow-up of liver injury and disease. The ALT enzyme plays an important role in amino acid metabolism and gluconeogenesis by catalyzing reversible transamination between L-alanine and $\alpha$-ketoglutarate to form pyruvate and glutamate. While the liver is the most ALT-enriched organ in the human body, ALT activity is also found in other organs, such as the heart, kidney and skeletal muscle (2). Two isoforms of ALT have been discovered, ALT1 and ALT2, which originate from 2 discrete genes $(3,4)$. Based upon the amino acid sequence of liver cytosolic ALT, the human glutamate pyruvate transaminase (GPT1) gene has been cloned and mapped to chromosome 8q24.3 (4). Yang et al (3) mapped another human ALT isozyme (GPT2), to chromosome 16q12.1. These respective proteins are both functional enzymes but with different cellular locations, with the ALT1 protein being present in the cytoplasm and the ALT2 protein in the mitochondria (5-11).

Traditionally, due to very low levels of ALT protein in plasma, the standard ALT assay measures total ALT enzymatic activity in serum or plasma. This method does not differentiate between the ALT isoforms. However, we have shown in a previous study that the ALT1 protein is enriched in human liver, kidney and skeletal muscle, whereas the ALT2 protein is mostly expressed in the heart, skeletal muscle, pancreas, adrenal gland and brain (12). More importantly, the human liver is an organ with very high levels of ALT1 and very low levels of ALT2, suggesting that the ALT isoform measurement in plasma may 
increase the specificity of the total ALT measurement. To the best of our knowledge, in a previous study, we demonstrated for the first time the presence of ALT2 in human plasma (5) and developed an immunoprecipitation (IP) method to quantify the respective isoform in human plasma $(5,12)$. The results from these cited studies based on a limited number of healthy volunteers, suggested that the ALT1 protein represents the majority of ALT in the circulation ( $>90 \%$ ) with the remainder being the ALT2 protein $(<10 \%)$. However, it is uncertain whether the relative contribution of the ALT1 and ALT2 protein/activity in plasma changes due to damage in specific human organs. Available data from the immunohistochemical distribution of ALT1 and ALT2 in human organs suggest that during ongoing hepatic damage the proportion of ALT1 increases further in plasma and ALT2 decreases. Conversely, the heart and skeletal muscle are organs which are enriched in ALT2 protein expression $(5,12)$. This suggests that the relative proportion of ALT1 to ALT2 in plasma may change during skeletal muscle injury resulting in an increase of ALT2 in plasma. Clinical chemical evidence is lacking for these suppositions due to the limited number of quantitative methods available for detecting ALT1 and ALT2 in human plasma.

Therefore, in the present study, we applied the isoformspecific ALT activity method developed in our laboratory to plasma from patients with ongoing liver damage resulting from non-alcoholic fatty liver disease (NAFLD), hepatitis C infection and hepatic surgery. We also analysed plasma from volunteers experiencing ongoing muscle injury after mixed ultra-endurance exercise (extended running, trekking and cycling). To our knowledge, our data provide the first indication that the ALT isoform activity changes in plasma depending upon the organ damage involved.

\section{Materials and methods}

\section{Subjects and study design}

NAFLD study. Ten healthy non-diabetic normal subjects (3 men and 7 women) and 9 NAFLD patients ( 3 men and 6 women) were recruited from the Department of Medicine at the University of Helsinki. Other inclusion criteria were: i) age 18-65 years; ii) no known acute or chronic disease, based on history, physical examination and standard laboratory tests (blood counts, serum creatinine, thyroid-stimulating hormone and electrolyte concentrations) and ECG; iii) alcohol consumption $<20$ g/day. The subjects did not use drugs known to affect liver fat content such as steroids, thiazolidinediones or vitamin E. The study protocol was approved by the Ethics Committee of the Helsinki University Central Hospital. The subjects were divided into 2 groups with either increased liver fat content in the absence of other causes [NAFLD, liver fat content $>5.5 \%$ measured using proton magnetic resonance spectroscopy $\left({ }^{1} \mathrm{H}-\mathrm{MRS}\right) \mathrm{n}=9$ ] (13) and with normal liver fat content $(n=10,<5.5 \%$ measured using ${ }^{1} \mathrm{H}-\mathrm{MRS}$ ) (14). Blood samples were taken after an overnight fast in citrate tubes and centrifuged within $30 \mathrm{~min}$ at room temperature and stored at $-80^{\circ} \mathrm{C}$ until they were batch-shipped to AstraZeneca, Södertälje, Sweden for the analysis of liver biomarkers.

Hepatitis C study. Twenty-three study subjects (17 men and 6 women) with chronic hepatitis $\mathrm{C}$ were recruited from the Hepatobiliary Clinic at the Mayo Clinic Rochester, MN, USA and compared to 20 (13 men and 7 women) normal subjects. Adult (age $>18$ years) patients evaluated for chronic hepatitis $\mathrm{C}$ infection were identified using the electronic medical records system. The presence of hepatitis $\mathrm{C}$ virus (HCV) infection was verified by positive HCV RNA in the serum. Non-liver disease control subjects were recruited from patients evaluated for esophageal or motility symptoms at the Mayo Clinic. Adult subjects (age $>18$ years) without a history of liver disease were identified. This study was approved by the Institutional Review Board of the Mayo Foundation. After an informed consent was obtained, study subjects underwent phlebotomy. Blood $(10 \mathrm{ml})$ was drawn into heparin tubes from which plasma was separated following a standard protocol. The plasma specimens were stored at $-80^{\circ} \mathrm{C}$ until they were batch-shipped to AstraZeneca, Södertälje, Sweden for analysis of liver biomarkers.

Liver surgery study. Included in this study at the Karolinska University Hospital (Gastro Center) at Huddinge, Sweden were 8 male and 4 female patients with a mean age of 66.6 ( $\mathrm{SD} \pm 11.6)$ years, undergoing resection of malignant tumors in the liver, including 1 primary and 9 secondary tumors, as well as 2 tumors of uncertain origin. The selection criteria were as follows: i) adult patient, ii) undergoing open liver resection and iii) scheduled for $>4 \mathrm{~h}$ of surgery. The study was approved by the Stockholm Region Ethics Review Board. Pre-operative body mass index (BMI) ranged from 18.4 to 31.0, with a mean of 25.9. The mean blood loss was 1.13 liters $(\mathrm{SD} \pm 0.95)$ and the mean length of hospital stay was $11.1(\mathrm{SD} \pm 2.9)$ days. Blood samples were drawn the morning of the surgery and subsequently at the same time on the first post-operative day. Samples of $4 \mathrm{ml}$ were drawn into EDTA tubes, centrifuged within $15 \mathrm{~min}$ and immediately frozen at $80^{\circ} \mathrm{C}$ until they were batch-shipped to AstraZeneca, Södertälje, Sweden for the analysis of liver biomarkers.

Extreme adventure race study. Thirty nine participants (8 women and 31 males) were recruited, based on the following inclusion criteria: i) well trained women and men with experience from adventure races longer than $24 \mathrm{~h}$, age 20-40 years; ii) no known acute or chronic disease based on history, physical examination and standard laboratory tests; iii) stated alcohol consumption $<20 \mathrm{~g} /$ day. The subjects did not use drugs known to affect physical performance; however, non-steroidal antiinflammatory drugs (NSAIDs) were used by the majority of the participants for pain management. In total, 8 women and 31 men performed mixed ultra-endurance exercise of running, trekking, kayaking, cycling and climbing in 3 different adventure races with average finishing race times of 48, 72 and $150 \mathrm{~h}$, respectively (15-18). Average energy expenditure including sleep and rest was $\sim 525 \mathrm{kcal} / \mathrm{h}$, corresponding to $\sim 40 \%$ of individual maximal oxygen uptake (18). Energy, water intake and time for rest were ad libitum. Before and within $20 \mathrm{~min}$ after the end of the race, blood samples were obtained in citrate tubes and centrifuged within $30 \mathrm{~min}$ at room temperature and stored at $-80^{\circ} \mathrm{C}$ until they were batch-shipped to AstraZeneca, for the analysis of liver biomarkers. The study protocols were approved by the Regional Ethics Review Board in Stockholm, Sweden.

NAFLD study: liver fat content $\left({ }^{1} H-M R S\right)$. Liver fat was measured as previously described (19). Three generations of 1.5 Tesla clinical scanners manufactured by Siemens 
(Sonata and Avanto) were used. Prior to MRS measurements, T1-weighted localization images were collected using a standard ${ }^{1} \mathrm{H}$ body coil. A $8-27 \mathrm{~cm}^{3}$ voxel was carefully positioned avoiding large vessels, bile ducts and the gall bladder. The subjects were allowed to breathe freely during the data collection. All spectra were analysed with the MRUI/jMRUI software using VARPRO/AMARES (20). The peak intensities resonating from the protons of the water and methylene [ $(\mathrm{CH} 2) n-2]$ groups in the fatty acid chains were determined using line shape fitting with prior knowledge. Signal intensities were corrected for T1 and T2 relaxation. Liver fat content was expressed as the ratio of the signal from the methylene group to the total signal of methylene and water. Liver fat content was converted from a signal ratio to a weight fraction applying the method validated by Longo et al (21) and Szczepaniak et al (14). The following experimentally determined factors were used: i) the ratio of the number of lipid protons in the fitted $(\mathrm{CH} 2) \mathrm{n}-2$ signal to the total number of lipid protons is 0.6332 ; ii) proton densities of fat and water; iii) $1 \mathrm{~g}$ of liver tissue contains $711 \mathrm{mg}$ water; iv) densities of the liver tissue, fat in the liver and water are 1.051,0.900 and $1.000 \mathrm{~g} / \mathrm{ml}$, respectively.

Antibodies and recombinant protein generation. The ALT1/ ALT2 antibodies generation, specificity and their use in western blot analysis, immunohistochemistry and IP in plasma have been described previously $(5,12)$. In these publications, we also described the cloning and the expression of 2 human GPT forms: GPT1 and GPT2. The purpose of generating recombinant proteins was for the validation of the IP assay on plasma. Enzymatic ALT activity from the purified recombinant proteins was measured according to the International Federation of Clinical Chemistry (IFCC) with pyridoxal phosphate activation (ALTLP: ACN 684, Cobas, Roche). Enriched recombinant proteins were stored at $-80^{\circ} \mathrm{C}$ in ALT buffer, containing phosphate-buffered saline (PBS), $1 \mathrm{mg} / \mathrm{ml}$ BSA and $25 \%$ glycerol, until further use.

Measurement of organ damage biomarkers in plasma. Measurement of relative levels of ALT1 and ALT2 in plasma was performed on ice or at $+4^{\circ} \mathrm{C}$. Plasma $(100 \mu \mathrm{l})$ was co-incubated with ALT1 antibodies (Ab.10995, $1.0 \mu \mathrm{g}$ ) end-over-end for $30 \mathrm{~min}$ at $4^{\circ} \mathrm{C}$. Dynabeads $(50 \mu \mathrm{l}, \mathrm{M}-280$ sheep anti-rabbit; Dynal, Invitrogen) were added to the plasma and incubated for an additional $30 \mathrm{~min}$, with end-over-end mixing. The Dynabeads were removed using a magnet and the remaining solution was used for ALT activity measurements (acc. IFCC, ALTLP: ACN 684, Cobas 501, Roche). The remainding ALT that was not precipitated by ALT1 antibody was defined as the ALT2 fraction (5). Calculations of ALT isoforms are specified below. The Cobas 501 analyser was used with standard protocols from Roche to measure alkaline phosphatase (ALP), aspartate aminotransferase (AST), $\gamma$-glutamyltransferase (GGT), glutamate dehydrogenase (GLDH), creatine kinase (CK), amylase, lipase, bilirubin and albumin in human plasma. We demonstrated that the lower limit of quantification (LLOQ) for the ALT assay using Cobas 501, Roche were <1 U/1 (data not shown). All individual plasma samples were measured once per subject and the means \pm SD from the absolute values were calculated on a group basis and presented in the tables with statistical analysis detailed below.
Statistical analyses and ALT calculations. ALT data generated from clinical chemistry measurement in plasma from the 4 groups in this study were treated by measuring individual values for total ALT in U/l. After IP with ALT1 antibody of human plasma, remeasurement of plasma samples for each individual provided the remaining ALT activity, which was defined as the ALT2 fraction (U/l). The calculation of ALT1 (U/l) was performed by subracting total ALT-ALT2 for each individual in each group. The percentage of ALT1 was calculated by dividing ALT1 by the total ALT x100 and the percentage of ALT2 was calulated by dividing ALT2 by the total ALT x100 for each individual. The ratio ALT1/ALT2 was calculated by dividing the ALT1 (U/l) value with the ALT2 (U/l) value for each individual. The means \pm SD for ALT, ALT1, ALT2, the percentage of ALT1, percentage of ALT2 and ALT1/ALT2 are presented in the respective tables. The unpaired Student's t-test was used to compare the mean values between the groups (NAFLD and hepatitis C). A paired t-test was used when comparing the same person before and after injury (liver surgery and adventure race study). A P-value $<0.05$ was considered to indicate a statistically significant difference. Correlation analyses were performed using Pearson's correlation. Calculations were performed using GraphPad Prism version 4.00 for Windows (GraphPad Software, San Diego, CA).

\section{Results}

Validation of ALT isoform assay. The IP assay of ALT1 from human plasma was evaluated for performance as regards capacity, reproducibility, LLOQ, specificity and stability. Some of these experiments have been described in our previous studies $(5,12)$. Since the amounts of ALT1 and ALT2 are very low in human plasma for western blot analysis detection, the measurement of ALT enzymatic activities (total ALT) has been the only way to quantify these proteins until the recent application of the mass spectrometry analysis (22). However, by combining IP and western blot analysis, we were able to detect both ALT1 and ALT2 proteins in a human plasma sample (5). The results of the additional validation of the ALT isoform assay are listed below. Capacity: Recombinant ALT1 was added to the human plasma samples at increasing concentrations (0-2,354 U/1) and total ALT activity was measured before and after IP with ALT1 antibody. The percentage ALT activity after IP was constant and varied between 2-6\% for ALT activities up to $715 \mathrm{U} / 1$. Adding ALT1 recombinant protein amounting to an excess of $715 \mathrm{U} / 1$ enzymatic activity, drastically increased the percentage of ALT activity after IP, showing the limit of the assay 700 U/1. Reproducibility: ALT1 was immunoprecipitated from 10 human plasma samples in duplicates by 2 different laboratory technicians. The remaining ALT activity in the plasma samples (ALT2) was measured using standard ALT activity assay (Cobas). The average activity after the first IP was $2.5 \pm 1.7$ and after the second IP was $2.7 \pm 1.9$, which was a non-significant difference $(\mathrm{P}=0.8)$ between the 2 experiments. LLOQ: $0.9 \%$ sodium chloride solution was primed with standard ALT (Cobas 501; Roche). In a typical experiment, repeated measurement of $\mathrm{NaCl}$ solution without ALT protein provide 0 , 0,0 and $0 \mathrm{U} / \mathrm{l}(\mathrm{n}=4)$ and for a solution with ALT protein provide $38,38,38$ and 38 . By dilution this ALT sample 1:10, provided readings of $4,4,4,4 \mathrm{U} / \mathrm{l}$ (return 105\%) and 1:20 dilution 
Table I. Values of liver injury markers in healthy controls vs. NAFLD patients.

\begin{tabular}{lcccc}
\hline Injury markers & Healthy controls & NAFLD & Fold-change & P-value \\
\hline ALT (U/l) & $17 \pm 7$ & $55 \pm 26$ & 3.2 & $<0.001$ \\
ALT1 (U/1) & $15 \pm 6$ & $52 \pm 25$ & 3.5 & $<0.001$ \\
ALT2 (U/1) & $1 \pm 1$ & $3 \pm 1$ & 3 & 0.004 \\
ALT1 (\%) & $92 \pm 5$ & $94 \pm 4$ & - & NS \\
ALT2 (\%) & $8 \pm 5$ & $6 \pm 4$ & - & NS \\
ALT1/ALT2 & $10.1 \pm 3.5$ & $18.7 \pm 9.2$ & 1.7 & 0.03 \\
AST (U/1) & $19 \pm 3$ & $36 \pm 11$ & 1.9 & $<0.001$ \\
AST/ALT & $1.2 \pm 0.4$ & $0.7 \pm 0.2$ & - & 0.003 \\
ALP (U/1) & $43 \pm 15$ & $49 \pm 19$ & 1.1 & NS \\
GGT (U/1) & $24 \pm 18$ & $44 \pm 26$ & 1.8 & NS \\
GLDH (U/l) & $1.8 \pm 0.4$ & $4.9 \pm 2.3$ & 2.5 & $<0.001$ \\
CK (U/l) & $100 \pm 47$ & $136 \pm 50$ & 1.4 & NS \\
\hline
\end{tabular}

NAFLD, non-alcoholic fatty liver disease; ALT, alanine aminotransferase; AST, aspartate aminotransferase; ALP, alkaline phosphatase; GGT, $\gamma$-glutamyltransferase; GLDH, glutamate dehydrogenase; CK, creatine kinase; NS, not significant.

provided 2, 1, 2, 2, 2, 2, $2 \mathrm{U} / 1$ (return 98\%). The LLOQ for the ALT assay using Cobas 501 using these protocols was $1 \mathrm{U} / 1$. The lower detection limit reported for ALT by the provider (Roche) is $5 \mathrm{U} / 1$, calculated as the value lying 3 standard deviations above the lowest standard (1 U/l). We examined whether lower values could be measured, and by diluting a positive control we achieved a good return and repeatablility down to 2 U/1. The LLOQ for the ALT assay using Cobas 501 using these protocols was $1 \mathrm{U} / 1$. Specificity: $1 \mu \mathrm{g}$ ALT1 antibody (with $50 \mu$ l Dynabeads) precipitated $100 \%$ of the ALT activity from the solution containing ALT1 recombinant protein with an activity of $106 \mathrm{U} / 1$. In a mixture of $50 \%$ recombinant ALT1 and ALT2, total activity was $130 \mathrm{U} / 1$ in which ALT1 theoretically contributes $\sim 53 \mathrm{U} / 1$ and ALT2 $77 \mathrm{U} / 1$. After IP with the ALT1 antibody, $69 \mathrm{U} / 1$ activity remained, indicating that the ALT1 antibody decreased by $>100 \%(115 \%)$ of the theoretical ALT1 contributing to the activity in the solution. Stability: we previously published data showing a rapid breakdown of ALT2 in $37^{\circ} \mathrm{C}$ and that both ALT1 and ALT2 were stable on ice $\left(+4^{\circ} \mathrm{C}\right)$ during these experimental conditions (5). Recombinant ALT1 and ALT2 remained stable in storage at $-80^{\circ} \mathrm{C}$ for 6 months; however, ALT2 was unstable at $-20^{\circ} \mathrm{C}$ and showed a loss of activity after 1 week of storage (data not shown).

NAFLD study. Plasma samples from 10 healthy controls and 9 NAFLD patients were compared in this study. The samples were selected based upon those with the largest difference in liver fat content between the 2 groups. The liver fat contents were $2.3 \pm 0.6 \%$ and $20.2 \pm 1.0 \%(\mathrm{P}<0.001)$ in the healthy controls and NAFLD patients, respectively. Other clinical characteristics between the controls and NAFLD patients, such as age $\left(52 \pm 3\right.$ and $50 \pm 3$ years), BMI $\left(32.3 \pm 1.9\right.$ and $\left.34.1 \pm 1.6 \mathrm{~kg} / \mathrm{m}^{2}\right)$ and waist circumference $(107 \pm 4$ and $111 \pm 5 \mathrm{~cm})$ did not differ between the groups.

The total ALT activity in the plasma was increased by 3.2 -fold $(\mathrm{P}<0.001)$ in the NAFLD group $(55 \pm 26 \mathrm{U} / \mathrm{l})$ compared to the normal subjects $(17 \pm 7 \mathrm{U} / 1)$ (Table I). The components of total ALT activity, ALT1 and ALT2, showed both statistically significant higher activity values, with $\sim 3$-fold $(\mathrm{P}<0.001)$ elevations in the NAFLD group compared to the control group. The percentage contributions of each isoform to the total ALT activity were $92 \%$ for ALT1 and $8 \%$ for ALT2 in the normal subjects and $94 \%$ for ALT1 and $6 \%$ for ALT2 in the NAFLD patients (not statistically significant). However, the ALT1/ALT2 ratio was significantly higher in the NAFLD group (18.7 \pm 9.2$)$ than in the control group $(10.1 \pm 3.5, \mathrm{P}<0.05)$. Analysis of liver and muscle injury markers, such as ALP, AST, CK, GGT and GLDH showed differences between the groups only for AST that were higher in the NAFLD group $(36 \pm 11)$ compared to the normal subjects $(19 \pm 3, \mathrm{P}<0.001)$ and GLDH was also higher in the NAFLD group $(1.8 \pm 0.4)$ compared to normal subjects $(4.9 \pm 2.3, \mathrm{P}<0.001)$. The ratio AST/ALT was $0.7 \pm 0.2$ in the NAFLD group and $1.2 \pm 0.4, \mathrm{P}=0.003$ in the normal subjects.

Hepatitis $C$ study. The total ALT activity was $~ 5$-fold higher in the hepatitis $\mathrm{C}$ group (117 U/l), when compared to the control group (23 U/1, P<0.001) (Table II). In parallel, the ALT1 activity was increased by $\sim 5$-fold in the hepatitis $C$ group as compared to the control group. The relative contribution of ALT1 was higher for the hepatitis $\mathrm{C}$ group $(96 \pm 4 \%)$ than for the control group $(93 \pm 3 \%, \mathrm{P}<0.01)$. The ALT2 activity, was only increased by 2.5 -fold in the hepatitis $\mathrm{C}$ group, with a relative contribution $(4 \pm 2 \%)$ in the hepatitis $\mathrm{C}$ group and $(7 \pm 3 \%, \mathrm{P}<0.01)$ in the control group. The ratio ALT1/ALT2 was increased from $12 \pm 6$ in the control group to $26 \pm 13(\mathrm{P}<0.001)$ in the hepatitis $\mathrm{C}$ group. Whilst the other liver injury markers, ALP, AST, GGT and GLDH, were also significantly elevated (ranging from 1.6- to 4.5-fold) compared to the healthy controls (Table II), there were no differences in the skeletal muscle marker and CK activity between the 2 groups.

Liver surgery study. This was an intervention-type study, in which 12 patients who underwent hepatic surgery to remove tumor tissue were examined for liver biomarkers before and after 
Table II. Values of liver and muscle injury markers in healthy controls vs. hepatitis C patients.

\begin{tabular}{lcccr}
\hline Injury markers & Healthy controls & Hepatitis C patients & Fold-change & P-value \\
\hline ALT (U/l) & $23 \pm 13$ & $117 \pm 83$ & 5.1 & $<0.001$ \\
ALT1 (U/l) & $21 \pm 12$ & $112 \pm 79$ & 5.3 & $<0.001$ \\
ALT2 (U/1) & $2 \pm 2$ & $5 \pm 5$ & 2.5 & 0.008 \\
ALT1 (\%) & $93 \pm 3$ & $96 \pm 4$ & - & 0.006 \\
ALT2 (\%) & $7 \pm 3$ & $4 \pm 2$ & - & 0.006 \\
ALT1/ALT2 & $12.1 \pm 6$ & $26.5 \pm 13$ & 2.2 & $<0.001$ \\
AST (U/1) & $27 \pm 6$ & $112 \pm 79$ & 4.1 & $<0.001$ \\
AST/ALT & $1.4 \pm 0.6$ & $1.1 \pm 0.5$ & - & NS \\
ALP (U/l) & $61 \pm 21$ & $98 \pm 31$ & 1.6 & $<0.001$ \\
GGT (U/l) & $21 \pm 12$ & $95 \pm 66$ & 4.5 & $<0.001$ \\
GLDH (U/1) & $2 \pm 1$ & $9 \pm 6$ & 4.5 & $<0.001$ \\
CK (U/l) & $136 \pm 131$ & $117 \pm 69$ & 0.9 & NS \\
\hline
\end{tabular}

ALT, alanine aminotransferase; AST, aspartate aminotransferase; ALP, alkaline phosphatase; GGT, $\gamma$-glutamyltransferase; GLDH, glutamate dehydrogenase; CK, creatine kinase; NS, not significant.

Table III. Circulating markers before and after liver surgery.

\begin{tabular}{|c|c|c|c|c|}
\hline Markers & Before surgery & After surgery & Fold-change & P-value \\
\hline ALT (U/l) & $31 \pm 20$ & $357 \pm 317$ & 11.5 & 0.003 \\
\hline ALT1 (U/l) & $29 \pm 19$ & $345 \pm 303$ & 11.9 & 0.003 \\
\hline ALT2 (U/1) & $2 \pm 1$ & $13 \pm 15$ & 6.5 & 0.03 \\
\hline ALT1 (\%) & $91 \pm 4$ & $97 \pm 2$ & - & $<0.001$ \\
\hline ALT2 (\%) & $9 \pm 4$ & $3 \pm 2$ & - & $<0.001$ \\
\hline ALT1/ALT2 & 12.8 & 37.7 & 2.9 & 0.002 \\
\hline $\operatorname{AST}(\mathrm{U} / \mathrm{l})$ & $41 \pm 20$ & $419 \pm 489$ & 10.2 & 0.016 \\
\hline AST/ALT & $1.5 \pm 0.6$ & $1.1 \pm 0.3$ & - & 0.02 \\
\hline $\operatorname{ALP}(\mathrm{U} / \mathrm{l})$ & $27 \pm 13$ & $19 \pm 7$ & 0.7 & NS \\
\hline Bilirubin $(\mu \mathrm{mol} / \mathrm{l})$ & $13 \pm 11$ & $23 \pm 21$ & 1.8 & 0.016 \\
\hline GLDH (U/l) & $7 \pm 5$ & $154 \pm 219$ & 22 & 0.032 \\
\hline GGT (U/l) & $81 \pm 84$ & $73 \pm 67$ & 0.9 & NS \\
\hline Lipase (U/l) & $38 \pm 20$ & $28 \pm 16$ & 0.7 & NS \\
\hline $\mathrm{CK}(\mathrm{U} / \mathrm{l})$ & $85 \pm 63$ & $933 \pm 664$ & 11 & $<0.001$ \\
\hline
\end{tabular}

ALT, alanine aminotransferase; AST, aspartate aminotransferase; ALP, alkaline phosphatase; GGT, $\gamma$-glutamyltransferase; GLDH, glutamate dehydrogenase; CK, creatine kinase; NS, not significant.

the surgery. The total ALT activity in plasma before surgery was $31 \pm 20 \mathrm{U} / 1$ and increased dramatically (11.5-fold) after hepatic surgery to $357 \pm 317 \mathrm{U} / 1, \mathrm{P}<0.01$ (Table III). The fold increase of total ALT activity ranged from 3.1 to 70.5 and correlated sufficiently with the surgeons estimated 'size' of the hepatic surgery (data not shown). While the absolute ALT1 activity in plasma increased from 29 to $345 \mathrm{U} / 1$ (11.9-fold, $\mathrm{P}<0.01)$, a similar but smaller increase (6.5-fold) was observed for ALT2, increasing in absolute terms, from 2 to $13 \mathrm{U} / 1$ (6.5-fold, $\mathrm{P}<0.05)$. However, despite the fact that the ALT2 activity increased in absolute terms, this translated to an actual decrease of the percentage of ALT2 in plasma from 9 to $3 \%(\mathrm{P}<0.001)$. The percentage distribution of ALT1, on the other hand, increased from $91 \%$ before surgery to $97 \%$ of total activity after surgery $(\mathrm{P}<0.001)$. The ratio ALT1/ALT2 increased from 13 to $38(\mathrm{P}<0.01)$ during liver surgery. The regression co-efficient for ALT to ALT1 for all samples was $1.0(\mathrm{P}<0.001)$, and for ALT to ALT2, $\mathrm{r}=0.92$ $(\mathrm{P}<0.0001)$. Various other liver injury markers as seen from Table III changed dramatically. GLDH activity, increased from $7 \pm 5 \mathrm{U} / 1$ before surgery to $154 \pm 219$ after surgery in agreement with the ALT data. However, the GLDH response to surgery varied greatly on an individual level, with fold increases ranging from 2.6- to 166.4-fold. The individual values for total ALT fold increase and GLDH fold increase correlated significantly 
Table IV. Circulating markers before and after adventure race.

\begin{tabular}{lcccc}
\hline Markers & Pre-race & Post-race & Fold-change & P-value \\
\hline ALT (U/l) & $18 \pm 6$ & $72 \pm 36$ & 4.0 & $<0.001$ \\
ALT1 (U/l) & $18 \pm 6$ & $66 \pm 32$ & 3.7 & $<0.001$ \\
ALT2 (U/l) & $1 \pm 1$ & $5 \pm 5$ & 5.0 & $<0.001$ \\
ALT1 (\%) & $96 \pm 4$ & $93 \pm 4$ & - & $<0.001$ \\
ALT2 (\%) & $4 \pm 4$ & $7 \pm 4$ & - & $<0.001$ \\
ALT1/ALT2 & $15.7 \pm 6$ & $18.9 \pm 14$ & - & NS \\
AST (U/l) & $28 \pm 8$ & $184 \pm 131$ & 6.6 & $<0.001$ \\
AST/ALT & $1.6 \pm 0.4$ & $2.5 \pm 0.7$ & 1.6 & $<0.001$ \\
ALT2/ALT & $0.04 \pm 0.04$ & $0.07 \pm 0.04$ & 1.8 & $<0.001$ \\
ALP (U/l) & $56 \pm 21$ & $59 \pm 21$ & 1.0 & NS \\
Bilirubin $(\mu$ mol/l) & $5 \pm 2$ & $10 \pm 5$ & 2.1 & $<0.001$ \\
Albumin (g/l) & $42 \pm 7$ & $42 \pm 3$ & 1.0 & NS \\
GGT (U/l) & $16 \pm 8$ & $16 \pm 6$ & 1.0 & NS \\
GLDH (U/l) & $4 \pm 2$ & $4 \pm 3$ & 1.0 & NS \\
CK (U/l) & $131 \pm 62$ & $3866 \pm 3327$ & 29.5 & $<0.001$ \\
\hline
\end{tabular}

ALT, alanine aminotransferase; AST, aspartate aminotransferase; ALP, alkaline phosphatase; GGT, $\gamma$-glutamyltransferase; GLDH, glutamate dehydrogenase; CK, creatine kinase; NS, not significant.

$(\mathrm{r}=0.81, \mathrm{P}<0.001)$. Additionally, AST increased by 10 -fold and the hepatobiliary liver injury marker, bilirubin, increased by 1.8-fold, while GGT and ALP activities were unchanged by liver surgery in these patients. The pancreatic amylase activity increased by 2.2 -fold, while the plasma lipase activity remained unchanged.

Adventure race study. This was an intervention-type study and involved 39 participants who performed mixed ultraendurance exercise with average finishing race times of 48 , 72 and $150 \mathrm{~h}$. The average age of the participants was $31 \pm 5$ years, with a mean body weight of $75.8 \pm 10.3 \mathrm{~kg}$ and a BMI of 23.6 \pm 1.8 . All data obtained before and after the race with finishing times of 48, 72 and $150 \mathrm{~h}$ were combined and used to calculate the mean values of the changes in the plasma biomarkers. Total ALT in the plasma increased by 4-fold from $18 \mathrm{U} / 1$ before the race to $72 \mathrm{U} / 1$ after the completion of the race (Table IV). The ALT1 isoform increased from 18 to $66 \mathrm{U} / 1$ and ALT2 from 1 to $5 \mathrm{U} / 1$. The percentage distribution of ALT isoforms decreased for ALT1 from 96 to $93 \%$ and increased for ALT2 from 4 to $7 \%$. The ratio of ALT1/ALT2 remained unchanged. The enzyme, AST, which is more of a muscle than a liver marker, increased more compared to ALT (6.6-fold). The more sensitive skeletal muscle marker, CK increased by 29.5-fold in response to the competition. The ratio of AST/ ALT was used to differentiate muscle from liver injury and a ratio $<1.0$ indicated greater liver injury than muscle injury. The ratio increased from 1.6 to 2.5 after the completion of the race. Using ALT2 as a putative skeletal muscle marker and substituting ALT2 for AST in the same ratio (AST/ALT becomes ALT2/ALT) also showed an increased ratio from 4.0 to 7.4, which is similar in order of magnitude as for AST/ ALT. Other liver injury markers, such as ALP, GGT, GLDH and albumin were unaffected by the physical efforts during the race. However, bilirubin was increased from $5 \mu \mathrm{mol} / 1$ before the race to $10 \mu \mathrm{mol} / 1$ after the race.

ALT, ALT1 and ALT2 in all controls and baseline samples. Previous data using small groups of healthy volunteers have demonstrated the distribution of ALT1 to be $~ 90 \%$ and that of ALT2 to be $\sim 10 \%$ in human plasma $(5,12)$. In the present study, we calculated the average ALT levels for all the control subjects in the 4 groups ( $n=81$ individuals). Total ALT was $20.8 \pm 11.3 \mathrm{U} / 1$ with ALT1 representing $19.4 \pm 10.5 \mathrm{U} / 1$ and ALT2 $1.4 \pm 1.3 \mathrm{U} / 1$. This amounted to $94 \%( \pm 4 \%)$ ALT1 and $6 \%$ $( \pm 4 \%)$ ALT2 in normal plasma. The ratio of ALT1/ALT2 was $13.5 \pm 6.5$ in normal individuals. Those individuals $(n=19)$ with an ALT2 value of 0 were not taken into account when calculating the average ratio ALT1/ALT2. In Table V, a summary is shown for ALT in healthy subjects and the changes that occurred in ALT isoforms during liver and skeletal muscle injury.

\section{Discussion}

This study is to our knowledge, the first that demonstrates that in humans with either liver or skeletal muscle damage, the proportion of the ALT isoenzymes changes in plasma reflecting leakage from a hepatic or an extrahepatic source. Total ALT activity is assayed extensively in both routine clinical practice and in the safety assessment of emerging pharmaceuticals as a surrogate marker for liver injury. The selectivity of this single biomarker works sufficiently as long as the injury to the liver is severe enough to signal large increases of ALT activity to the plasma or serum (23). Despite the fact that ALT is widely viewed as a specific indicator to liver necrosis, ALT activity is found in a number of other organs such as the kidney, heart, skeletal muscle and pancreas (2). Damage to any of these organs 
Table V. Summary of ALT profiles from healthy subjects, during liver damage (surgery) and during skeletal muscle damage (adventure race).

Total ALT (U/1)

Percentage ALT1

Percentage ALT2

\begin{tabular}{lccr}
\hline Healthy subjects $^{\mathrm{a}}$ & 21 & $94 \%$ & $6 \%$ \\
Liver damage $^{\mathrm{b}}$ & $31 \rightarrow 357$ & $91 \rightarrow 97 \% \Uparrow$ & $9 \rightarrow 3 \% \Downarrow$ \\
Skeletal muscle damage $^{\mathrm{c}}$ & $18 \rightarrow 72$ & $96 \rightarrow 93 \% \Downarrow$ & $4 \rightarrow 7 \% \Uparrow$ \\
\hline
\end{tabular}

ALT, alanine aminotransferase; ${ }^{a} \mathrm{n}=81$ individuals; ${ }^{\mathrm{b}} \mathrm{n}=12$ individuals before and after liver surgery; ${ }^{\mathrm{c}} \mathrm{n}=39$ individuals before and after the adventure race (skeletal muscle damage).

also releases ALT into the blood, making ALT measurement less specific for liver injury (24-26). Over a 10-fold increase in basal ALT activity by skeletal muscle injury alone has been observed during controlled conditions (25). Further complicating this is the fact that ALT is not a single enzyme, but consists of 2 isoforms, ALT1 and ALT2, both with similar enzymatic capacity (5). We and others have shown that the distribution of these ALT isoforms differs between tissues and organs $(3,5,12)$. The liver and kidney are particularly abundant in ALT1 expression, with almost no detectable ALT2, whereas the heart and skeletal muscle express both ALT1 and ALT2. Therefore, it has been postulated that the isoform-specific measurement of ALT in serum may provide additional information as regards extrahepatic organ damage than total ALT activity alone $(3,12)$. More importantly, if both ALT1 and ALT2 are present in plasma, and are released from different organs during damage, whether or not total ALT activity is wrongly interpreted depends on a battery of additional biomarkers being measured simultaneously. Another concern involves the change from baseline ALT during an intervention ingestion of a pharmaceutical with possible effects on organ damage. The proportion change (percentage or ratio) of ALT1 to ALT2 may reflect the different organ damage (i.e. hepatic injury having one profile and extrahepatic injury, such as skeletal muscle having another).

In this study, we developed an isoform-specific ALT assay and applied it to human plasma samples from patients having liver or skeletal muscle injury. The first group in the population of liver injury included a small set of patients diagnosed with a fatty liver. The second group in this population comprised patients diagnosed with hepatitis $\mathrm{C}$ and the third a group undergoing liver surgery to remove metastatic cancer tissue (intervention study). All these groups have a common thread of different degrees of injury to the liver. The second population (also intervention study) included subjects performing ultra-endurance exercise lasting 48,72 or $150 \mathrm{~h}$ in which the purpose was to study ALT isoforms in a setting of muscle injury with no degree of liver injury. Traditional markers for skeletal muscle and liver injury were also incorporated in the measurements apart from ALT isoforms in order to ensure the specificity for respective organ damage studied.

Total serum ALT activity was increased in all 4 groups. Thus, in the NAFLD patients, ALT was 3-fold elevated over the healthy controls, whereas in hepatitis $\mathrm{C}$-infected patients a 5-fold elevated activity was noted in comparison to the healthy controls. In the intervention experiments, after liver surgery the same patient had on average $>11$-fold increase in plasma ALT activity after completion of the surgery on the same day. Interestingly, patients who removed the spleen in the same clinic did not have an increase of total ALT, indicating that the surgical procedure did not affect the ALT values (data not shown). GLDH has been suggested to be a more specific and sensitive biomarker for liver injury in mainly preclinical studies but its clinical use is limited (27). In this study, GLDH was elevated 2.5-fold in NAFLD patients and 4.5-fold in hepatitis $\mathrm{C}$ patients, both correlating well with the increase of ALT. However, during direct surgery, GLDH was elevated 22-fold but with a larger variation (SD) than corresponding total ALT activities. Therefore, from these limited populations our data suggest the assay of serum GLDH to be at least as sensitive as the traditional ALT measurement for detecting liver injury in humans. By contrast, the assay of GGT, known to detect damage to the biliary tracts in the liver, was not increased significantly in the NAFLD patients. On the other hand, in hepatitis C-infected patients, GGT was increased by 4.5-fold as compared to the healthy controls. Interestingly, GGT was unchanged during liver injury induced by surgery. These data suggest that GGT is not a specific marker for hepatocyte cell death as ALT and GLDH, which is in line with the present knowledge of GGT. In a similar fashion, serum ALP, which is also considered a marker for biliary damage and for bone diseases, was only elevated in hepatitis C-infected patients (1.6-fold increase over controls). In addition, serum bilirubin, also an established marker for hepatobiliary damage and known to be the most specific but least sensitive general liver injury biomarker, was only increased after liver surgery (1.8-fold over baseline). By contrast, serum CK, a biomarker of skeletal muscle damage, was unchanged in NAFLD and hepatitis C-infected patients, compared to their respective healthy controls, indicating that the observed increases in AST and ALT arose from hepatic origin. However, in our study population that underwent liver surgery, there was a large increase (11-fold over baseline) in serum CK. This effect is known to occur immediately after surgery. Patients who had only their spleen removed during the same surgical procedures displayed $\mathrm{CK}$ increases (on average 8-fold, $\mathrm{n}=3$ ) (data not shown). The reasons for this effect are not fully understood. However, it has been suggested that the cause may be either from the procedure of cutting muscle tissues to reach the organ of interest or the anaesthetics used during the surgery (28).

Since GLDH was not expressed in skeletal muscle tissue and was increased by 22 -fold after surgery, it may be assumed 
that most of the ALT change observed was due to liver injury. In support of this, was the ratio of AST/ALT in plasma which is often used to implicate the degree of skeletal and liver damage $(26,29)$. Before surgery the AST/ALT ratio was 1.5 , with a decrease of the ratio to 1.1 after surgery, implying liver damage. Interestingly, an AST/ALT ratio below 1.0 has been associated with liver conditions, such as fatty liver and hepatitis (29). In this study, the ratio AST/ALT was 0.65 in NAFLD patients and 0.95 in hepatitis $C$ patients. The ratios of AST/ALT in the control groups were 1.1 and 1.2 for NAFLD and the hepatitis $\mathrm{C}$ group, respectively. The absolute numbers of activity of ALT1 and ALT2 increased in all 3 liver injury experiments and the percentage distribution of ALT1 and ALT2 over the total ALT changed in all clinical groups in the same direction; the percentage of ALT1 in plasma increased during liver disease or surgery of the liver, while the percentage of ALT2 decreased with the same percentage number. This is interpreted as follows: since the liver is an organ with a higher ALT1 than ALT2 expression, upon hepatocyte damage more ALT1 than ALT2 is released in the blood. Alternatively, the ratio of ALT1/ALT2 also reflects the same observations using percentage distribution in these conditions. The ALT1/ALT2 ratio increased significantly in all 3 clinical groups of liver injury.

Interestingly, in the 4th experiment of skeletal muscle injury, total ALT activity increased 4-fold. Studying the absolute numbers of ALT1 and ALT2 does not provide additional information regarding the difference between liver surgery and skeletal muscle injury. However, the percentage ALT1 decreased from $96 \%$ before the race, to $93 \%$ after the race. At the same time, ALT2 increased from $4 \%$ to $7 \%$ after completing the race. The ALT1/ALT2 ratio showed no statistically significant change by this intervention. Thus the relative proportion of ALT1 and ALT2 proceeds in the opposite direction during a liver vs. skeletal muscle injury. To our knowledge this has not been reported before. One would have expected a decrease in the ALT1/ALT2 ratio due to percentage changes of ALT1 and ALT2. We speculate that this is possibly due to skeletal muscle being an organ containing both ALT1 and ALT2 and the ratio does not show a decrease but instead an unchanged ratio. Thus, looking at fold changes of ALT1 and ALT2 together with percentage changes provides important information of hepatic or extrahepatic injury. The other biomarkers in this ultra race study indicate extreme skeletal muscle injury with or without liver injury (GLDH unchanged) despite a 4-fold increase in total ALT activity. The skeletal muscle marker, CK increased by almost 30-fold and AST by 6.6-fold with completion of the race. As stated previously, the ratio of AST/ALT was used to differentiate muscle from liver injury, which increased from 1.6 to 2.5 after the completion of the race, implicating the additional release of muscle AST into the circulation. Using ALT2 as a putative skeletal muscle marker and substituting ALT2 for AST in the same ratio (ALT2/ALT) also showed an increased ratio from 4.0 to 7.4 which is of the same magnitude as for AST/ALT. Hence, the measurement of ALT2 does not provide additional information compared to AST and CK as skeletal muscle markers in regards to sensitivity.

At the same time, GLDH, GGT and ALP were not changed by the race. On the other hand, bilirubin levels increased in the plasma (2.1-fold), which may indicate damage to liver biliary epithelial cells. An argument against this is the lack of changes in GGT and ALP levels. The most plausible explanation is the fact that several participants consumed pain relievers (NSAIDS) during the adventure race (30). NSAIDS are competing for the same transporters, OATP1B1, into the liver as bilirubin, which could explain the increased bilirubin levels in the plasma (31).

In conclusion, both ALT1 and ALT2 activities may be quantified in human plasma in healthy controls and in patients suffering from fatty liver disease or hepatitis $C$. These patients have higher total ALT activity in the plasma mainly due to increased levels of ALT1, presumably being released from the affected organ (liver). To determine this, patients undergoing liver surgery as a model for a more controlled liver injury recorded increased total ALT activity, with an increased percentage of ALT1 and a decreased percentage of ALT2. By contrast, volunteers performing adventure races with extreme physical exertion, also have higher total ALT activities in plasma; however, the ALT isoform proportion was reversed compared to liver injury. The percentage of ALT1 decreased and the percentage of ALT2 increased in plasma during muscle injury/breakdown. This separate analysis of ALT1 and ALT2, being the sum component of total ALT activity, has to our knowledge not been previously performed using clinical samples (Table V). For screening purposes, measuring the percentage contribution of ALT1 and ALT2 in plasma in addition to the total ALT enzyme activity, may offer an opportunity to understand whether extrahepatic injury (pancreas, heart, adrenal gland, skeletal muscle, intestine, etc.) occurs in cases where total ALT levels may falsely be interpreted as liver injury. Therefore, our data suggest that this crude distinction may be made without any other organdamage biomarkers other than the ALT proteins themselves. Besides existing novel data, this study displays 2 important findings: specifically during liver injury, the measurement of total ALT activity is sufficient on its own, since the liverspecific ALT1 correlates perfectly with total ALT activity in the plasma. Additionally, specifically during skeletal muscle injury, the measurement of ALT2 activities using the IP method is sufficient, as putative skeletal muscle has approximately the same sensitivity as AST, but less sensitivity compared to CK measurement. Future research is required in order to demonstrate whether the measurement of ALT1 and ALT2 proteins, not their enzymatic activities, will add additional value to the currently established clinical chemistry damage markers.

\section{References}

1. Karmen A, Wroblewski F and Ladue JS: Transaminase activity in human blood. J Clin Invest 34: 126-131, 1955.

2. Lindena J, Sommerfeld U, Hopfel C and Trautschold I: Catalytic enzyme activity concentration in tissues of man, dog, rabbit, guinea pig, rat and mouse. Approach to a quantitative diagnostic enzymology, III. Communication. J Clin Chem Clin Biochem 24: 35-47, 1986.

3. Yang RZ, Blaileanu G, Hansen BC, Shuldiner AR and Gong DW: cDNA cloning, genomic structure, chromosomal mapping, and functional expression of a novel human alanine aminotransferase. Genomics 79: 445-450, 2002.

4. Sohocki MM, Sullivan LS, Harrison WR, et al: Human glutamate pyruvate transaminase (GPT): localization to 8q24.3, cDNA and genomic sequences, and polymorphic sites. Genomics 40: 247-252, 1997.

5. Glinghammar B, Rafter I, Lindstrom AK, et al: Detection of the mitochondrial and catalytically active alanine aminotransferase in human tissues and plasma. Int J Mol Med 23: 621-631, 2009. 
6. DeRosa G and Swick RW: Metabolic implications of the distribution of the alanine aminotransferase isoenzymes. J Biol Chem 250: 7961-7967, 1975.

7. Pette D and Luh W: Constant-proportion groups of multilocated enzymes. Biochem Biophys Res Commun 8: 283-287, 1962.

8. Meton I, Egea M, Fernandez F, Eraso M C and Baanante IV: The N-terminal sequence directs import of mitochondrial alanine aminotransferase into mitochondria. FEBS Lett 566: 251-254, 2004.

9. Swick RW, Barnstein PL and Stange JL: The metabolism of mitochondrial proteins. I. Distribution and characterization of the isozymes of alanine aminotransferase in rat liver. J Biol Chem 240: 3334-3340, 1965.

10. Gubern G, Imperial S, Busquets $M$ and Cortes A: Partial characterization of the alanine aminotransferase isoenzymes from human liver. Biochem Soc Trans 18: 1288-1289, 1990

11. Gubern G, Imperial S, Busquets M and Cortes A: Subcellular distribution of alanine aminotransferase activity in human liver. Biochem Soc Trans 18: 1287-1288, 1990.

12. Lindblom P, Rafter I, Copley C, et al: Isoforms of alanine aminotransferases in human tissues and serum - differential tissue expression using novel antibodies. Arch Biochem Biophys 466 : 66-77, 2007.

13. Neuschwander-Tetri BA and Caldwell SH: Nonalcoholic steatohepatitis: summary of an AASLD Single Topic Conference. Hepatology 37: 1202-1219, 2003.

14. Szczepaniak LS, Nurenberg P, Leonard D, et al: Magnetic resonance spectroscopy to measure hepatic triglyceride content: prevalence of hepatic steatosis in the general population. Am J Physiol Endocrinol Metab 288: E462-E468, 2005.

15. Mattsson CM, Stahlberg M, Larsen FJ, Braunschweig F and Ekblom B: Late cardiovascular drift observable during ultraendurance exercise. Med Sci Sports Exerc 43: 1162-1168, 2011.

16. Berg U, Enqvist JK, Mattsson CM, et al: Lack of sex differences in the IGF-IGFBP response to ultra endurance exercise. Scand J Med Sci Sports 18: 706-714, 2008

17. Wallberg L, Mikael Mattsson C, Enqvist JK and Ekblom B: Plasma IL-6 concentration during ultra-endurance exercise. Eur J Appl Physiol 111: 1081-1088, 2011.

18. Enqvist JK, Mattsson CM, Johansson PH, Brink-Elfegoun T, Bakkman L and Ekblom BT: Energy turnover during $24 \mathrm{~h}$ and 6 days of adventure racing. J Sports Sci 28: 947-955, 2010.
19. Kotronen A, Peltonen M, Hakkarainen A, et al: Prediction of non-alcoholic fatty liver disease and liver fat using metabolic and genetic factors. Gastroenterology 137: 865-872, 2009.

20. Vanhamme L, van den Boogaart A and Van Huffel S: Improved method for accurate and efficient quantification of MRS data with use of prior knowledge. J Magn Reson 129: 35-43, 1997.

21. Longo R, Pollesello P, Ricci C, et al: Proton MR spectroscopy in quantitative in vivo determination of fat content in human liver steatosis. J Magn Reson Imaging 5: 281-285, 1995.

22. Miyazaki M, Rosenblum JS, Kasahara Y, Nakagawa I and Patricelli MP: Determination of enzymatic source of alanine aminotransferase activity in serum from dogs with liver injury. J Pharmacol Toxicol Methods 60: 307-315, 2009.

23. Aithal GP, Watkins PB, Andrade RJ, et al: Case definition and phenotype standardization in drug-induced liver injury. Clin Pharmacol Ther 89: 806-815, 2011.

24. Giesen PL, Peltenburg HG, de Zwaan C, Janson PC, Flendrig JG and Hermens WT: Greater than expected alanine aminotransferase activities in plasma and in hearts of patients with acute myocardial infarction. Clin Chem 35: 279-283, 1989.

25. Barshes NR, Lee TC, Goodpastor SE, et al: Transaminitis after pancreatic islet transplantation. J Am Coll Surg 200: 353-361, 2005.

26. Pettersson J, Hindorf U, Persson P, et al: Muscular exercise can cause highly pathological liver function tests in healthy men. $\mathrm{Br}$ J Clin Pharmacol 65: 253-259, 2008.

27. O'Brien PJ, Slaughter MR, Polley SR and Kramer K: Advantages of glutamate dehydrogenase as a blood biomarker of acute hepatic injury in rats. Lab Anim 36: 313-321, 2002.

28. Phornphutkul KS, Anuras S, Koff RS and Seeff LB: Causes of increased plasma creatine kinase activity after surgery. Clin Chem 20: 340-342, 1974.

29. Giannini EG, Testa R and Savarino V: Liver enzyme alteration: a guide for clinicians. CMAJ 172: 367-379, 2005.

30. Wichardt E, Mattsson CM, Ekblom B and Henriksson-Larsen K: Rhabdomyolysis/myoglobinemia and NSAID during $48 \mathrm{~h}$ ultraendurance exercise (adventure racing). Eur J Appl Physiol 111: 1541-1544, 2011

31. Ah YM, Kim YM, Kim MJ, et al: Drug-induced hyperbilirubinemia and the clinical influencing factors. Drug Metab Rev 40 511-537, 2008. 